\title{
INFLUÊNCIA DO đÊNERO NA PERCEPÇÃO EMOCIONAL DE USUÁRIOS DE CADEIRAS DE RODAS A PARTIR DO AUTORRELATO E WICROEXPRESSÃO FACIAL
}

\author{
GENDER INFLUENCE ON EMOTIONAL PERCEPTION \\ OF WHEELCHAIR USERS BASED ON SELF-REPORT \\ AND FACIAL MICRO-EXPRESSION
}

Recebido em: 12 de janeiro de 2021

Aprovado em: 20 de março de 2021

Sistema de Avaliação: Double Blind Review

RCO|a. 13 | v. 2 | p. 73-87| mai./ago. 2021

DOI: https://doi.org/10.25112/rco.v2i0.2520

\section{Jamille Noretza de Lima Lanutti jamille_lanutti@hotmail.com}

Doutora em Design, com ênfase em Desenvolvimento de Produto e Ergonomia pela Universidade Estadual Paulista Júlio de Mesquita Filho (Bauru/Brasil). Professora Adjunta I do Departamento de Design na Universidade Federal do Rio Grande do Norte (Natal/Brasil).

\section{Douglas Daniel Pereira dougdanielpereira@gmail.com}

Doutor em Design na linha de pesquisa Desenvolvimento de Produto pela Universidade Estadual Paulista Júlio de Mesquita Filho (Bauru/Brasil).

\section{Fausto Orsi Medola fausto.medola@unesp.br}

Doutor em Ciências pela Universidade de São Paulo (São Paulo/Brasil).

Professor na Universidade Estadual Paulista Júlio de Mesquita Filho (Bauru/Brasil).

\section{Luis Carlos Paschoarelli luis.paschoarelli@unesp.br}

Livre-docente em Design Ergonômico pela Universidade Estadual Paulista (Bauru/Brasil).

Professor Titular na Universidade Estadual Paulista (Bauru/Brasil). 


\section{CONHECIMENTO ONLINE}

ISSN: $2176-8501$

\section{RESUMO}

A pessoa com deficiência é socialmente caracterizada por suas limitações e pelos dispositivos assistivos. Em especial a Cadeira de rodas (CR) é uma Tecnologia Assistiva vista como socialmente limitante. Isto pode influenciar a experiência emocional com estes produtos estigmatizados. Este estudo buscou compreender como o gênero influencia a percepção emocional de usuários de CR a partir da análise de duas diferentes ferramentas metodológicas: autorrelato (EMOG) e fisiológica (FaceReader ${ }^{\circledR}$ ). Participaram do estudo 20 usuários de CR, sendo 10 do gênero masculino e 10 do gênero feminino (idade média de 41,76 anos e dp. 11,34). Assim, foi possível qualificar e quantificar a emoção desses usuários, e os resultados alcançados com o presente estudo permitem afirmar que tanto o Emog quanto o FaceReader ${ }^{\circledR}$ identificam que os participantes do gênero masculino demonstram emoções mais positivas e com maior intensidade, se comparado aos participantes do gênero feminino.

Palavras-chave: Design Emocional. Cadeira de rodas. Microexpressão facial.

\section{ABSTRACT}

People with disabilities are socially characterized by their limitations and assistive devices. In particular, the Wheelchair (CR) is an Assistive Technology seen as socially limiting. This can influence the emotional experience with these stigmatized products. This study sought to understand how gender influences the emotional perception of CR users from the analysis of two different methodological tools: self-report (EMOG) and physiological (FaceReader ${ }^{\circledR}$ ). Twenty CR users participated in the study, 10 male and 10 female (mean age 41.76 years and SD 11.34). Thus, it was possible to qualify and quantify the emotion of these users, and the results achieved with the present study allow us to state that both Emog and FaceReader ${ }^{\circledR}$ identify that male participants demonstrate more positive emotions and with greater intensity, when compared to participants of the female gender.

Keywords: Emotional Design. Wheelchairs. Facial Micro-expression. 


\section{CONHECIMENTO}

\section{INTRODUÇão}

O estudo das relações entre o Design e a emoção, embora exija ferramentas e métodos específicos para ser analisada e compreendida, pode contribuir para o desenvolvimento e uma maior aceitação de produtos frequentemente associados a estigma negativo, tal como a Cadeira de Rodas (CR). De fato, a análise desta relação depende da compreensão dos fatores que influenciam a percepção de usuários de $\mathrm{CR}$, como por exemplo, os gêneros feminino e masculino, os quais deveriam ser considerados nos parâmetros projetuais visando comunicar valores positivos para o usuário.

Diferentes autores estudaram emoção e o design das CRs, buscando compreender que fatores influenciam o uso e o autoconceito de quem usa este dispositivo assistivo (COSTA et al., 2012; PAPE et al., 2012; CARNEIRO et al, 2015; MORDAK et al., 2017; MALLIN et al., 2015, DESMET et al, 2003; MAIA et al., 2010). Particularmente no que tange o "gênero" dos usuários, Costa et al. (2012), Lanutti et al. (2015) e Mordak et al. (2017) realizaram abordagens que apontam este fator como determinante para a percepção emocional da CR. Este apontamento é bastante relevante, visto que permite compreender a emoção evocada por artefatos negativamente estigmatizados; e gerar parâmetros de projeto com base em uma variável - o gênero - que está relacionada aos usuários e à sua diversidade.

Costa et al. (2012) realizaram um estudo visando identificar os principais fatores na relação entre a CR e seus usuários. Participaram 114 sujeitos, de ambos os gêneros, usuários e não usuários de CR. Foram empregados protocolos com base em 14 emoções descritas por Desmet e Dijkhuis (2003). Os resultados apontaram a influência dos fatores estética, idade, nível de instrução, gênero e atividade (atuação) profissional na percepção emocional das CRs. Para esses autores, a CR representa socialmente um símbolo explícito da deficiência.

O estudo de Lanutti et al. (2015) investigou a influência do gênero nas percepções dos usuários sobre o significado de sua própria CR. Participaram do estudo dez usuários de CR (05 do gênero masculino e 05 do gênero feminino), os quais responderam um protocolo baseado em Diferencial Semântico (TULLIS; ALBERT, 2008), composto por 20 pares de adjetivos relacionados a aspectos práticos e simbólicos. De modo geral, os resultados indicam que as percepções dos homens foram mais positivas que as das mulheres, tanto em função dos aspectos práticos, quanto simbólicos. Os autores discutiram que tais diferenças podem revelar problemas subjacentes que as usuárias enfrentam no uso diário de cadeiras de rodas e as consequências sociais disso. Já o estudo desenvolvido por Mordak et al (2017), teve como objetivo conhecer a influência de características demográficas, de gênero e tipo de deficiência na percepção emocional de CR, com usuários na Argélia. Participaram do estudo 74 usuários de CR (48 do gênero masculino e 26 do gênero feminino), que avaliaram suas próprias CR utilizando a Geneva Emotion Wheel 


\section{CONHECIMENTO}

(SCHERER, 2015), de modo analógico ou digital. Os resultados indicaram que as respostas emocionais dos usuários de CR variaram em termos de emoções e intensidade de emoção, com destaque ao tipo de deficiência e ao gênero.

Em todos esses estudos foram empregadas ferramentas metodológicas de autorrelato, as quais apresentam limitações. Reijneveld et al. (2003) apontam que o uso de artefatos pode evocar emoções de baixa intensidade, as quais são imperceptiveis para este tipo de ferramentas. Já Schlemmer (2012), reitera as limitações do autorrelato, ao afirmar que eles capturam apenas os estados emocionais conscientes dos usuários, sendo longos o suficiente para haver mudanças em termos emocionais ao longo da aplicação.

Para além disso, já existem disponíveis outras ferramentas aplicadas em estudos de percepção de emoção, em que se empregam as medições fisiológicas que, de acordo com Wensveen (1999), surgem das alterações que ocorrem nas atividades do sistema nervoso autônomo (SNA) e que são responsáveis pelas medidas das emoções no contexto fisiológico. Como exemplo se destacam: a eletromiografia facial usada por Boxtel (2010); a avaliação de resposta verbal considerando a entonação, velocidade, pausas e intensidade vocal (JOHNSTONE et al., 2000), visto que a voz se altera em função das emoções (IIDA, 2016); a resposta galvânica da pele, a qual varia de acordo com o estado emocional das pessoas (MANDRYK; ATKINS; INKPEN, 2006; LAPARRA-HERNANDEZ et al. 2009; LIU; LIU; LAI, 2014); e as microexpressões faciais descritas por Ekman (1975) e aplicadas por Zaman e Shrimpton-Smith (2006), Terzis et al. (2010); e Goldberg (2012) com auxílio do 'FaceReader' - Noldus ${ }^{\circledR}$; entre outros.

Diante deste contexto, o objetivo do presente estudo foi compreender como o gênero influencia a percepção emocional de usuários de cadeira de rodas - artefato estigmatizado - a partir da análise de duas diferentes ferramentas metodológicas: autorrelato (EMOG) e fisiológica (FaceReader ${ }^{\circledR}$ ).

\section{MATERIAIS E MÉTODOS}

\subsection{CARACTERÍSTICAS DO ESTUDO E ASPECTOS ÉTICOS}

O presente estudo caracteriza-se com uma abordagem transversal e exploratória. Por envolver a participação de usuários de CR, o protocolo foi aprovado pelo sistema CONEP-MS (CAAE 59717916.5.0000.5663). Todos os participantes foram esclarecidos dos objetivos do estudo e assinaram um Termo de Consentimento Livre e Esclarecido (TCLE). 


\section{CONHECIMENTO ONLINE}

ISSN: 2176-8501

\subsection{AMOSTRAGEM}

Participaram do estudo 20 usuários de CR, sendo 10 do gênero masculino e 10 do gênero feminino, com idade média de 41,76 anos (dp. 11,34), todos brasileiros. Os critérios de inclusão foram: usuários de cadeiras de rodas, adultos, com a percepção cognitiva preservada e pleno controle de atividades nos membros superiores. Os critérios de exclusão foram: participantes não alfabetizados e controle de tronco deficitário.

\subsection{OBJETO DE ANÁLISE}

No presente estudo foram considerados objetos de análise 04 (quatro) diferentes modelos de CRs de propulsão manual: CR A - Modelo 132 - marca Ortometal, com estrutura de aço carbono dobrável, assento e encosto em poliéster, apoios de pés retráteis e apoio de braços fixos (Figura 1a); CR B - Modelo AVD - marca Ortobras, assento e encosto estofado em nylon acolchoado, apoios de pés desmontável e apoio de braços rebatível (Figura 1b); CR C - Modelo StarLite - marca Ortobras, com estrutura de alumínio dobrável monobloco, assento rígido e encosto flexível, apoios de pés em forma de U com plataforma, sem apoio de braços e com protetor de roupa em nylon com abas (Figura 1c); CR D - Modelo Le Parkour marca Jumper, com estrutura de alumínio dobrável monobloco, colorida, assento e encosto ajustável por velcro, apoios de pés em forma de U com plataforma, sem apoio de braços e com protetor de roupa em nylon com abas (Figura 1d).
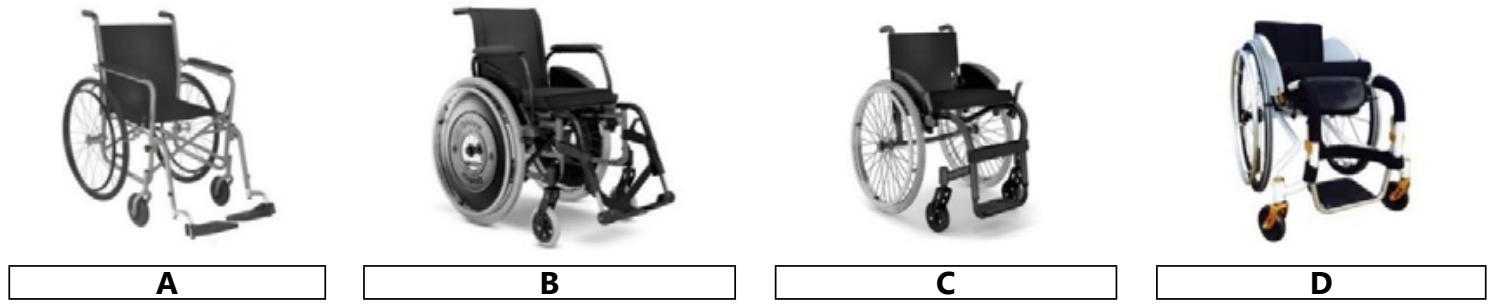

Figura 1 - Modelos de CR avaliados.

\subsection{FERRAMENTAS E PROCEDIMENTOS - EMOG}

O EMOG é uma ferramenta de autorrelato digital projetada para obter respostas da percepção emocional das pessoas diante de um estímulo, no caso particular a imagem de um produto (LANUTTI et al., 2015). Esta ferramenta baseia-se na avaliação de 20 (vinte) diferentes emoções, descritas nos estudos de Desmet e Dijkhuis (2003) e lida (2016) (Tabela 1). 


\section{CONHECIMENTO ONLINE}

ISSN: 2176-8501

\begin{tabular}{cc}
$\begin{array}{c}\text { Tabela 01: Tabela com as emoções utilizadas no Emog. } \\
\text { Emoções Positivas }\end{array}$ & Tristeza \\
\hline Alegria & Nojo \\
Desejo & Decepção \\
Inspiração & Desprezo \\
Admiração & Insatisfação \\
Satisfação & Rejeição \\
Aceitação & Tédio \\
Interesse & Desgosto \\
Gosto & Raiva \\
Fascínio & Medo \\
Surpresa & \\
\hline
\end{tabular}

Fonte: Os autores.

Sua aplicação ocorre em 03 (três) etapas: inicialmente o participante preenche cadastro de identificação (figura 2a); em seguida, emoções e imagens são apresentadas randomicamente e para cada emoção apresentada (com sua respectiva descrição) são também apresentadas 09 (nove) imagens para que o participante indique aquela que melhor representa àquela emoção (figura 2b); na terceira etapa são apresentadas imagens das CRs e, ao lado, as 20 (vinte) imagens representativas de cada uma das emoções selecionadas na etapa anterior, assim o participante indica apenas uma imagem (representativa daquela emoção) e quantificava sua intensidade em uma escala de 01 (um) a 06 (seis) (figura 2c).

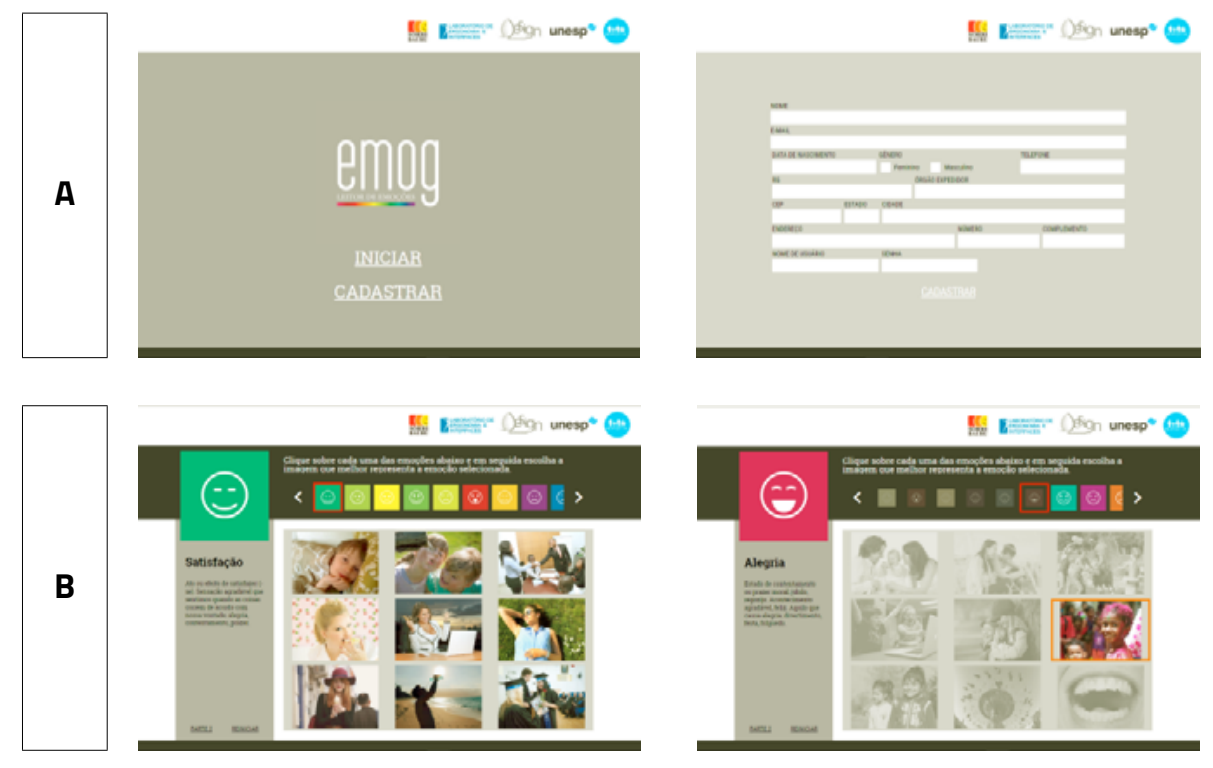




\section{CONHECIMENTO ONLINE}

ISSN: 2176-8501

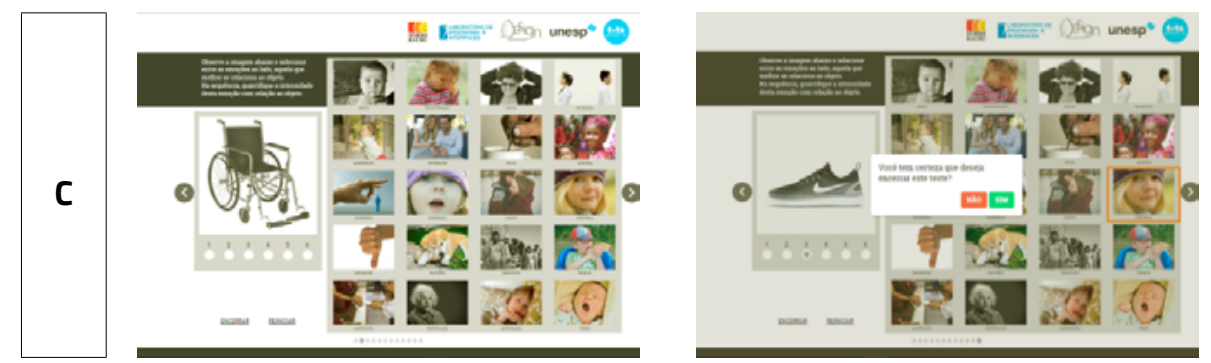

Figura 02 Telas de interface do Emog.

A análise dos dados ocorreu após a tabulação em planilha eletrônica (MicrosoftExcel ${ }^{\circledR}$ ), apresentando para cada CR quais emoções foram indicadas (na terceira etapa); quantas vezes foram indicadas; e qual a intensidade, descrita em valores de média e desvio padrão.

\subsection{FERRAMENTAS E PROCEDIMENTOS - FACEREADER}

O FaceReader ${ }^{\circledR}$ é uma ferramenta fisiológica digital desenvolvida para avaliar a percepção emocional a partir da variação das microexpressões faciais dos sujeitos, diante da apresentação de um estímulo (imagem ou vídeo), o qual foi registrado e analisado pelo software (NOLDUS, 2017).

Para o presente estudo, os participantes observaram vídeos (Figura 3), no qual um usuário de CR experiente realizou um percurso em um trajeto de curvas e retas, com cada uma das CRs. Com auxílio do software Camtasia $9{ }^{\circledR}$, tanto o monitor com o vídeo que estava sendo exibido quanto as faces dos participantes foram registrados (Figura 4), permitindo a análise posterior por meio do FaceReader ${ }^{\circledR}$.

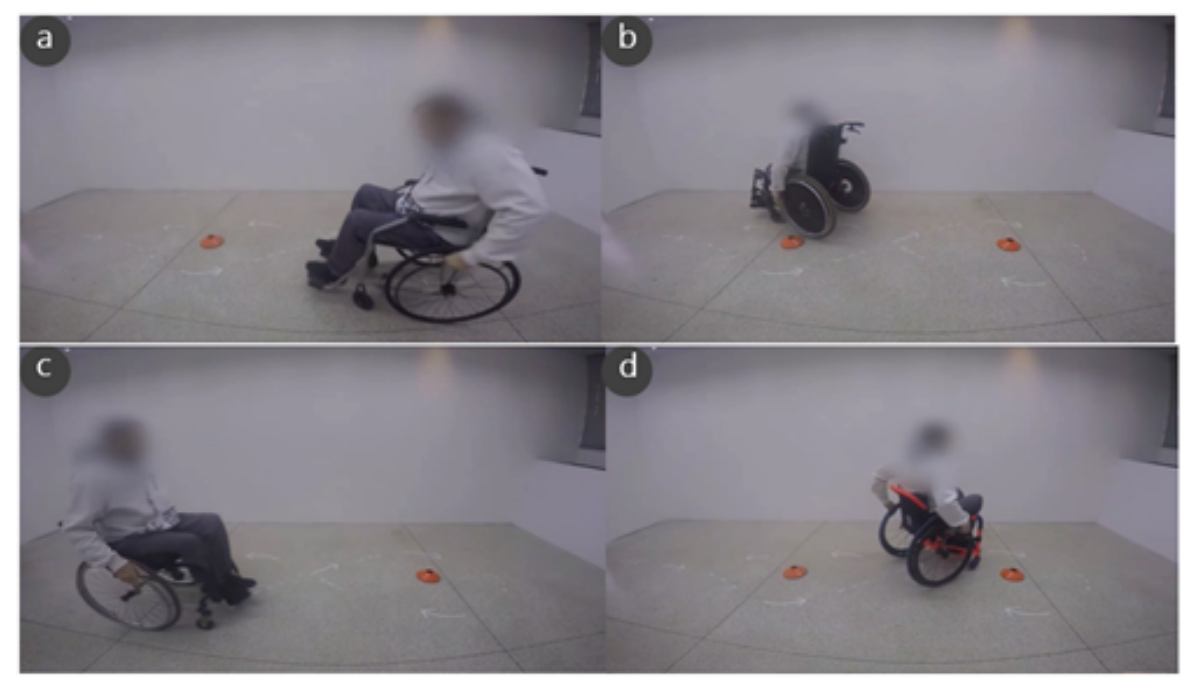

Figura 3 - Vídeo com trajeto sendo percorrido com os quatro modelos de CRs. 


\section{CONHECIMENTO ONLINE}

ISSN: 2176-8501

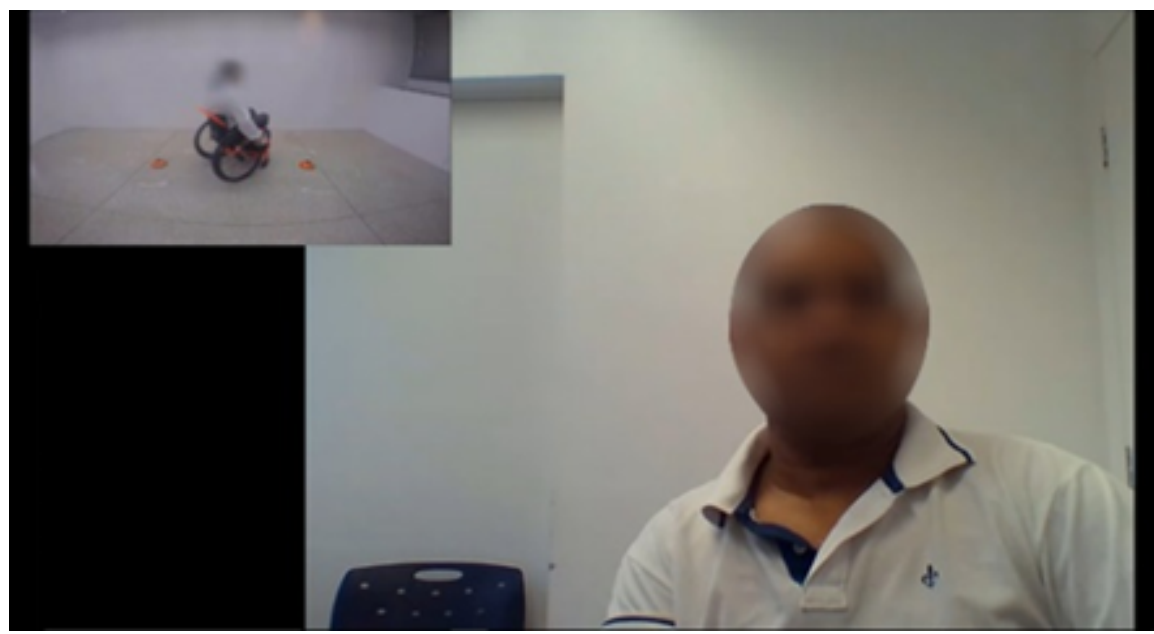

Figura 4 - Exemplo de como o vídeo para análise no FaceReader ${ }^{\circledR}$ foi capturado.

A análise dos dados obtidos com o FaceReader ${ }^{\circledR}$ ocorreu no próprio software. Para isso, cada um dos participantes do estudo teve seus dados previamente cadastrados, incluindo o gênero relatado. 0 software realizou a análise para cada parte do vídeo que aparece uma distinta CR, permitindo comparar as intensidades das emoções observadas nesses intervalos e para cada participante. Os resultados foram apresentados de forma descritiva (média e desvio padrão); e as análises comparativas realizadas por meio do teste $T$ de Student $(p \leq 0.05)$ - determinado pelo próprio software.

\section{RESULTADOS}

Os resultados da ferramenta de autorrelato digital Emog são apresentados na Figura 05. Observase que os participantes do gênero feminino selecionaram a mesma quantidade de emoções positivas e negativas (07 - sete), enquanto que os participantes do gênero masculino selecionaram mais emoções positivas (08 - oito) que negativas (06 - seis).

As emoções positivas 'Desejo,' 'Interesse', Inspiração', 'Aceitação', 'Satisfação', 'Alegria' e 'Gosto' são citadas por participantes de ambos os gêneros, contudo para os participantes do gênero masculino todas as emoções positivas alcançam médias de intensidade acima de 4,00.

Da mesma forma, os participantes de ambos os gêneros selecionam as emoções negativas 'Tédio', 'Decepção', 'Medo', 'Desgosto' e 'Insatisfação', contudo as médias de intensidade de todas as emoções negativas dos participantes do gênero feminino estão acima de 4,00. 


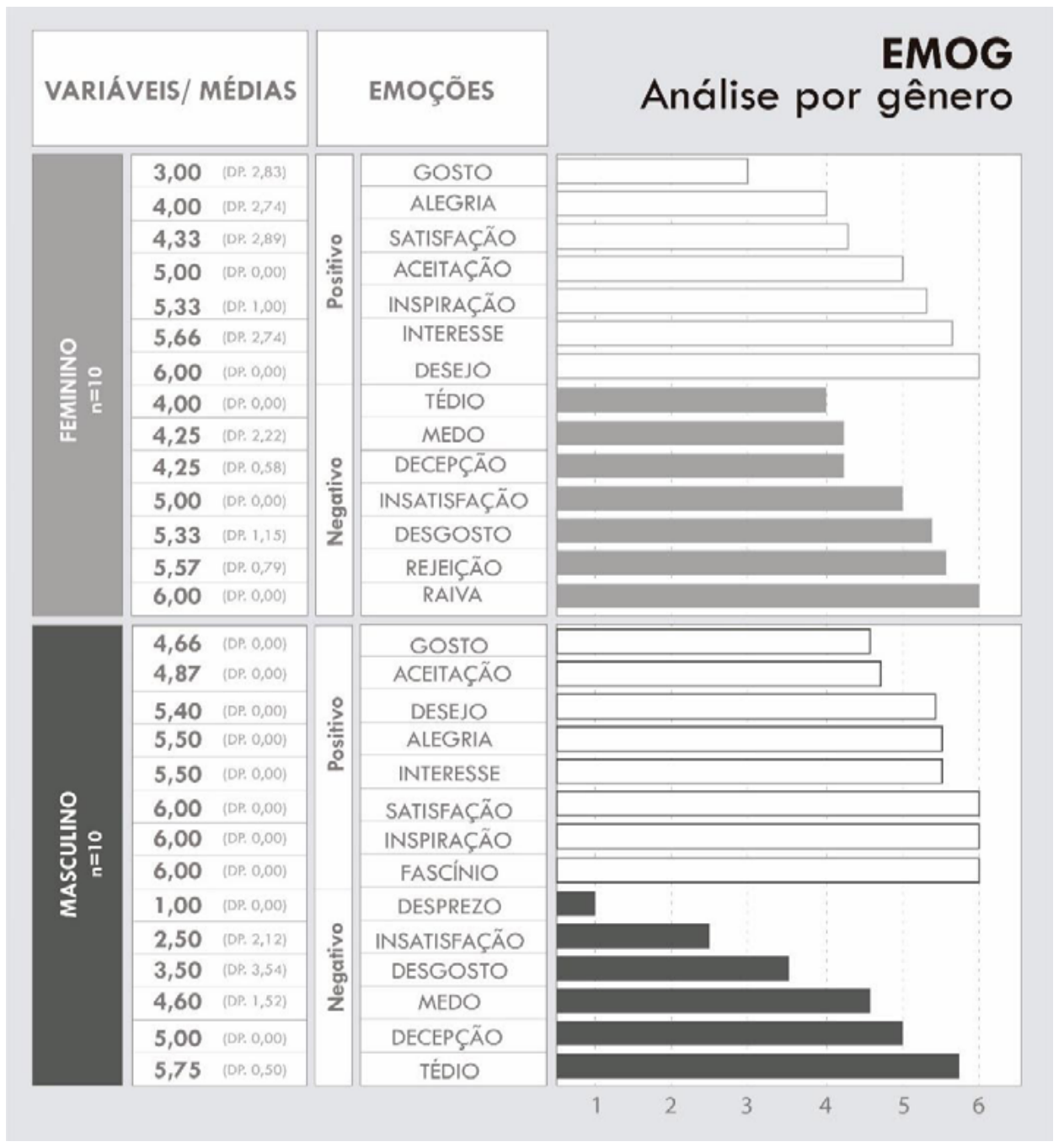

Figura 5 - Resultados para cada emoção registrada pelo Emog.

Das emoções que se diferem para os dois gêneros, os participantes do gênero masculino selecionaram a emoção negativa 'Desprezo' $(\square$ 1,00) e a emoção positiva 'Fascínio' $(\square$ 6,00). Já os participantes do gênero feminino selecionam as emoções negativas 'Rejeição' e 'Raiva', com médias 5,00 e 6,00, respectivamente.

Assim, observa-se que, para os participantes do gênero masculino, as emoções relacionadas a CR são mais positivas do que para os do gênero feminino, tanto na quantidade de emoções positivas selecionadas, quanto para o valor atribuído a emoção.

Os resultados obtidos com a ferramenta fisiológica FaceReader ${ }^{\circledR}$ são apresentados na Figura 06. 


\section{CONHECIMENTO ONLINE}

ISSN: 2176-8501

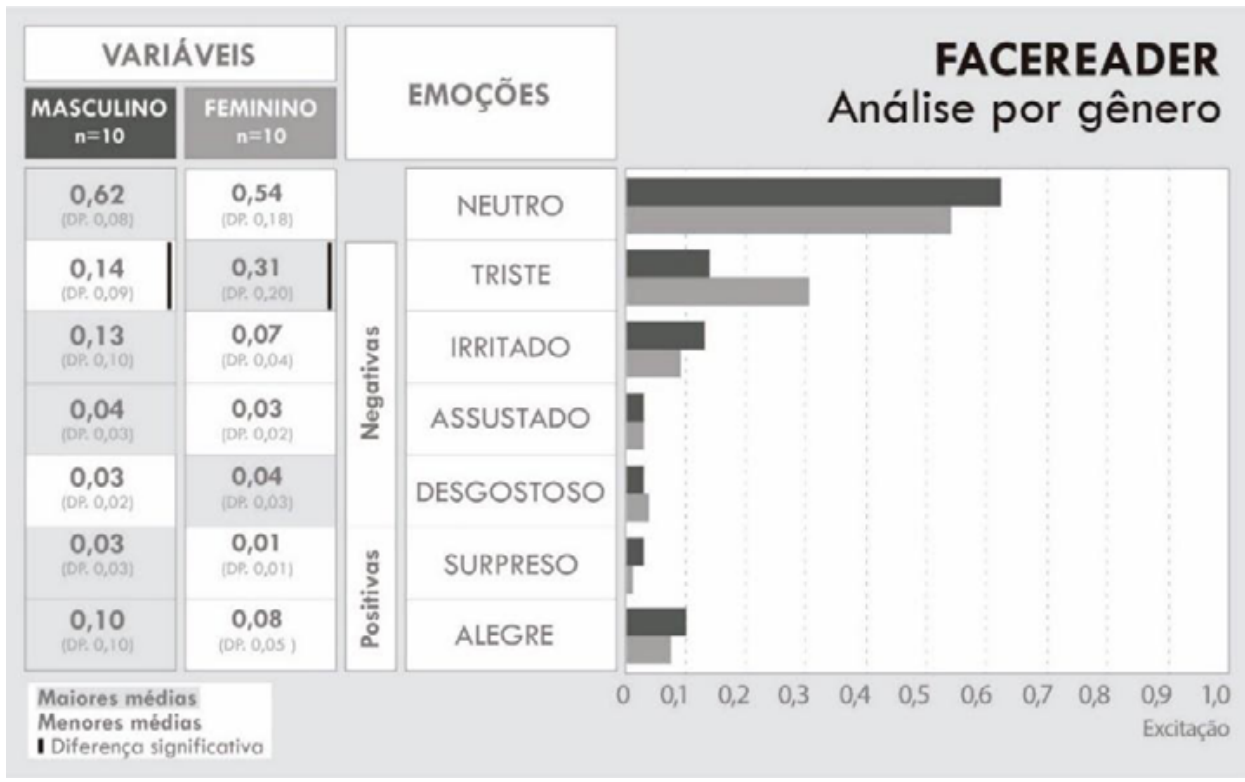

Figura 06 - Resultados para cada emoção registrada pelo FaceReader ${ }^{\circledR}$.

Esses resultados indicam que a condição "Neutro" apresentou maior média para os participantes do gênero masculino ( $\bar{X}$ 0,62; d.p. 0,08), se comparado aos resultados dos participantes do gênero feminino $(\bar{X} 0,54 ;$ d.p. 0,18), apesar de não haver diferença significativa entre os grupos ( $p>0,05)$. Os participantes do gênero masculino obtiveram as maiores médias de leituras faciais para a maioria das emoções avaliadas,

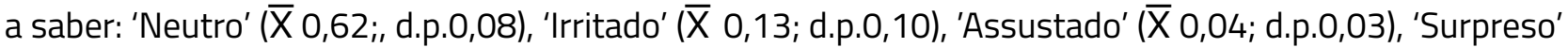

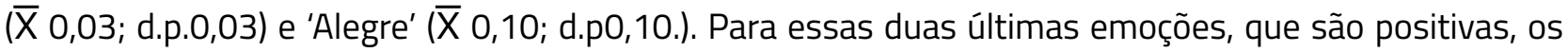
participantes do gênero masculino obtiveram maiores médias, se comparado com os participantes do gênero feminino ('Surpreso' = $\bar{X} 0,01 ;$ d.p.0,01 e 'Alegre' = $\bar{X} 0,08 ;$ d.p. 0,05). Já os participantes do gênero

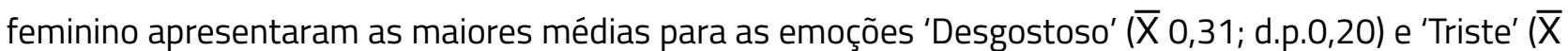
0,31 ; d.p.0,03), sendo que para a emoção 'Triste' a diferença é estatisticamente significativa $(p \leq 0,05)$, quando comparado aos participantes do gênero masculino.

\section{DISCUSSÃo}

A CR é um artefato de Tecnologia Assistiva associado a estigmas; e compreender os fatores que envolvem a relação artefato x usuário possibilita aos designers desenvolver alternativas que comuniquem valores positivos de e para seus usuários. 0 presente estudo propôs aplicar duas diferentes ferramentas 


\section{CONHECIMENTO}

metodológicas, EMOG (autorrelato) e FaceReader ${ }^{\circledR}$ (fisiológica), para compreender como os gêneros, masculino e feminino, dos usuários de CR influencia sua percepção emocional sobre o artefato.

Os resultados permitem afirmar que tanto o Emog (ferramenta de autorrelato) quanto o FaceReader ${ }^{\circledR}$ (ferramenta fisiológica) identificam que os participantes do gênero masculino demonstram emoções mais positivas e com maior intensidade, se comparado aos participantes do gênero feminino.

No que tange o fator gênero, os resultados reforçam aqueles descritos por Lanutti et al. (2015), os quais apontam que as usuárias de CR - gênero feminino - apresentam percepções mais negativas com relação às suas cadeiras de rodas, o que pode estar associado a sua imagem estética, refletindo em consequências sociais que reafirmam o estigma do artefato durante suas atividades. Também em relação ao gênero, Mordak et al. (2017) encontraram que a CR é um artefato que atrai o estigma para suas usuárias; enquanto que para os usuários - gênero masculino - a CR é o principal artefato de conexão com a sociedade, pois permite a locomoção, o acesso ao trabalho, ao estudo, enfim ao meio social externo. Costa e Moreira da Silva (2012) discutem a interferência do gênero na percepção emocional das CRs, que igualmente ajudam a compreender os resultados do presente estudo.

De fato, esses autores apontam que os aspectos técnicos - aqueles que ajudam a criar o gatilho para percepção emocional nas CRs - é mais valorizado pelos usuários do gênero masculino, especialmente quando se relaciona a um aumento de estabilidade e, consequentemente de segurança da CR (visto que a função de locomoção é primordial para esses usuários). Isto parece ser bastante coerente já que, de modo geral, o maior interesse dos usuários - gênero masculino - ocorre ao desempenho dos sistemas operacionais de um artefato (p.e.: o elevado desempenho energético de um equipamento doméstico - lava louça eletrônica), em oposição às usuárias - gênero feminino - que irão se interessar mais aos sistemas estéticos do mesmo produto (p.e.: o acabamento e a cor deste equipamento doméstico e sua relação com o ambiente que estará instalado - a cozinha planejada) e sua imagem social. Esta diferença pode ser resultado do que Baron-Cohen (2003) descreveu como uma melhor capacidade de sistematização do gênero masculino, que se relaciona a relevância dos aspectos técnicos, e uma maior capacidade de empatia do gênero feminino, que se relaciona a importância dos aspectos sociais.

Os resultados aqui apresentados permitem entender que, sendo a CR um artefato de mobilidade, cujos requisitos projetuais se concentram em oferecer maior estabilidade e segurança, parece sensato afirmar que, ao oferecer essas 'qualidades' aos usuários, aqueles do gênero masculino irão percebê-las muito mais positivamente, enquanto que as usuárias - gênero feminino - não encontram elementos que poderiam ser percebidos como positivos, mas apenas um complemento à função primária de locomoção e, portanto, o estigma inerente deste produto seria mantido e transferido para essas usuárias. 


\section{CONHECIMENTO}

Apesar dos estudos neste campo utilizarem exclusivamente ferramentas de autorrelato (COSTA et al. 2012; LANUTTI et al. 2015; e MORDAK et al. 2017), no presente estudo foram aplicadas diferentes ferramentas metodológicas - EMOG (autorrelato) e FaceReader ${ }^{\circledR}$ (fisiológica). Os resultados do FaceReader ${ }^{\circledR}$ - particularmente a emoção "tristeza" - apontam diferença significativamente maior para o gênero feminino, o que parece corroborar com os resultados do EMOG, não especificamente para esta emoção, mas para seus aspectos negativos. Terzis et. al (2010) destacam a eficiência do FaceReader ${ }^{\circledR}$, enquanto que Lewinski et. al (2015) reiteram sua expressiva confiabilidade na avaliação de emoções básicas.

Entretanto, de acordo com Zaman e Shrimpton-Smith (2006), é necessária uma combinação do FaceReader ${ }^{\circledR}$ com outros métodos de avaliação, o que foi aplicado no presente estudo e cujos resultados demonstram elevada coerência. Para além disto, considerando que o FaceReader ${ }^{\circledR}$ é uma ferramenta fisiológica, Caicedo (2006) destaca que este tipo de ferramenta apresenta limitações: incertezas sobre as associações entre reações fisiológicas e emoções específicas; elevados recursos humanos e financeiros; entre outros.

\section{CONCLUSÃO}

O design de CRs deve considerar a importância, para seus usuários, dos valores associados ao artefato em questão. No presente estudo, aplicando duas diferentes ferramentas metodológicas (EMOG - autorrelato; e FaceReader ${ }^{\circledR}$ fisiológica), notou-se que os diferentes gêneros (masculino e feminino) apresentam diferentes percepções emocionais sobre este mesmo artefato, o que foi corroborado por outros estudos.

Portanto, podemos afirmar que o design de CR deve "ultrapassar" os requisitos da função primária deste artefato; e complementar aquilo que já é amplamente estudado e desenvolvido pelas áreas da reabilitação e da engenharia. De fato, pensar em design de CR é pesquisar e desenvolver alternativas que possam realmente criar valores agregados ao artefato, especialmente se houver vínculo e identificação com seus usuários, sendo o "gênero" um fator preponderante para esta atenção. Provavelmente, as usuárias de CR demonstrarão emoções mais positivas e com maior intensidade, se esses artefatos não foram apenas "estáveis" e "seguros" (o mínimo que se espera habitualmente), mas que apresentem elementos que ativem o gatilho para percepções emocionais positivas, podendo estar descritas na aplicação de diferentes cores; acabamento; acessórios; funções; entre outros. A personalização no design e configuração destes dispositivos pode ser um caminho neste sentido. Além disto, e aproveitando a 


\section{CONHECIMENTO ONLINE}

ISSN: 2176-8501

discussão deste fator, novos estudos devem considerar também as diferentes identidades de gênero que podem interferir na percepção emocional de CRs. No presente estudo, também podemos afirmar que as ferramentas aplicadas apresentaram resultados coerentes entre si e sugerem que todos os estudos no campo emocional possam considerar - de modo complementar - a aplicação de procedimentos metodológicos de autorrelato e fisiológicos.

\section{AGRADECIMENTOS}

O presente estudo foi desenvolvido com apoio da CAPES - Processo 88887.095645/2015-013693/2014; CNPq - Processo 304619/2018-3 e Sorri Bauru.

\section{REFERÊNCIAS}

BARON-COHEN, S. A diferença essencial. Rio de Janeiro: Objetiva, p. 15-45, 2003.

BOXTEL, A. V. Facial EMG as a Tool for Inferring Affective States. In: 7th International Conference on Methods and Techniques in Behavioral (Measuring Behavior 2010), Eindhoven, p. 104-108, 2010.

CAICEDO, D. G. How do you feel? An assessment of existing tools for the measurement of Emotions and their application in consumer products research. Delft University of Technology. Department of Industrial Design. The Netherlands, 2009.

CARNEIRO, L.; REBELO, F.; FILGUEIRAS, E.; NOBREGA, P. Usability and User Experience of Technical Aids for People with Disabilities? A Preliminary Study with a Wheelchair. Procedia Manufacturing, 3 ( 2015) 6068 - 6074. 2015.

COSTA, P.; MOREIRA DA SILVA, F.; FIGUEIREDO, C. Function versus emotion in a wheelchair design. In: REBELO, F.; SOARES, M. (eds.). Advances in Cognitive Ergonomics. pp. 573-580, [USA], Taylor \& Francis Group, New York, ISBN: 978-1-4665-6055-0, 2012.

DESMET, P. M. A.; DIJKHUIS E. A wheelchair can be fun: a case of emotion-driven design. In: Proceedings of The International Conference on Designing Pleasurable Products and Interfaces, June 23-26, Pittsburgh, Pennsylvania, USA. The Association for Computing Machinery. 2003. 


\section{CONHECIMENTO ONLINE}

ISSN: $2176-8501$

EKMAN, P.; FRIESEN, W. Facial Action Coding System: A Technique for the Measurement of Facial Movement. Consulting Psychologists Press, 1975.

GOLDBERG, J. H. Relating perceived web page complexity to emotional valence and eye movement metrics. Proceedings of the Human Factors and Ergonomics Society 56th Annual Meeting, 501-505. 2012.

IIDA, l.; GUIMAÃES, L. B. M. Ergonomia: projeto e produção. 3. ed. São Paulo: Blucher, 2016.

LEWINSKI, P.; DEN UYL, T.; BUTLER, C. Automated facial coding: validation of basic emotions and FACS AUs. In: facereader - Journal of Neuroscience, Psychology and Economics 7, 227-236 (2014). Disponivel em: <https://doi.org/10.1037/npe0000033>. Acesso em:

LANUTTI, J. N. L.; MEDOLA, F. O.; GONÇALVES, D. D.; SILVA, L. M. da; NICHOLL, A. R. J.; PASCHOARELLI, L. C. The significance of manual wheelchairs: a comparative study on male and female users. Procedia Manufacturing, 3 (2015) 6079 - 6085. 2015.

LAPARRA-HERNÁNDEZ, J.; BELDA-LOIS, J. M.; MEDINA, E.; CAMPOS, N.; POVEDA, R. EMG and GSR signals for evaluating user's perception of different types of ceramic flooring. International Journal of Industrial Ergonomics, Volume 39, Issue 2, 2009, Pages 326-332, ISSN 0169-8141. Disponível em: <https://doi.org/10.1016/j.ergon.2008.02.011>. Acesso em:

LIU, F.; LIU, G.; LAI, X. "Emotional Intensity Evaluation Method Based on Galvanic Skin Response Signal". 2014 Seventh International Symposium on Computational Intelligence and Design. Hangzhou, 2014, pp. 257-261. Disponivel em: <https://10.1109/ISCID.2014.145>. Acesso em:

MAIA, F.; NIEMEYER, L.; FREITAS, S. F. de. A relação entre indivíduos com deficiência, suas emoções e o design de objetos de tecnologia assistiva. Anais do $9^{\circ}$ Congresso Brasileiro de Pesquisa e Desenvolvimento em Design. São Paulo, 2010.

MANDRYK, R. L.; ATKINS, M. S.; INKPEN, K. M. A continuous and objective evaluation of emotional experience with interactive play environments. $\mathrm{CHI}$ '06: Proceedings of the SIGCHI Conference on Human Factors in Computing Systems. April 2006 Pages 1027-1036. Disponivel em: <htt ps://10.1145/1124772.1124926>. Acesso em: 


\section{CONHECIMENTO ONLINE}

ISSN: 2176-8501

MORDAK, M.; MEBARKI, B.; BOUABDELLAH, L.; MOKDAD, L. I. Emotional Responses of the Disabled Towards Wheelchairs. Advances in Affective and Pleasurable Design, pp. 86-96, 2017.

MALLIN, S. S. V.; CARVALHO, H. G. Assistive Technology and User-Centered Design: emotion as element for innovation. Procedia Manufactoring, v. 3, p. 5570-5578, 2015.

PAPE, T.; KIM, J.; WEINER, B. The shaping of individual meanings assigned to assistive technology: a review of personal factors. Disability and rehabilitation, vol. 24, nº 1/2/3, pp. 5 - 20. 2002.

SCHERER, K. R. What are emotions? And how can they be measured? Social Science Information, v. 44, n. 4, p. 695-729, 2015.

SCHLEMMER, A. A emoção no design: uma discussão sobre as práticas e abordagens. $\mathbf{2}^{\circ}$ GAMPI PLURAL, 2012, Univille, Joinville, SC. 2012.

REIJNEVELD, K.; DE LOOZE, M.; KRAUSE, F.; DESMET, P. Measuring the Emotions Elicited by Office Chairs. International conference on designing pleasurable products and interfaces - Pittsburgh. Nova York: ACM Press, 2003. p. 6-10. 2003.

TERZIS, V.; MORIDIS, C. N.; ECONOMIDES, A. A. Measuring Instant Emotions During a Self-Assessment Test: The Use of FaceReader. Proceedings of Measuring Behavior 2010, Eindhoven, The Netherlands, August 24-27, pp. 192-195. 2010.

TULLIS, T.; ALBERT, W. Measuring the User Experience: Collecting, Analysing, and Presenting Usability Metrics. Burlington: Morgan Kaufmann, 2008.

WENSVEEN, Stephan A. G. Probing Experiences. Procedings of the first international conference on design \& emotion. [S.I.] 1999.

ZAMAN, B.; SHRIMPTON-SMITH, T. The FaceReader: measuring instant fun of use. NordiCHI '06:

Proceedings of the 4th Nordic conference on Human-computer interaction: changing roles, October, 2006, Pages 457-460. Disponivel em: <https://doi.org/10.1145/1182475.1182536>. Acesso em: 